\title{
Characterization of a Laser-Induced Plasma Using Time-Resolved Dual-Frequency-Comb Spectroscopy
}

\author{
Yu Zhang ${ }^{1,2}$, Caroline Lecaplain ${ }^{2}$, Reagan R. D. Weeks ${ }^{2}$, Jeremy Yeak $^{3}$, Sivanandan \\ S. Harilal ${ }^{4}$, Mark C. Phillips ${ }^{4}$, R. Jason Jones ${ }^{2}$ \\ 1. Department of Physics, University of Arizona, 1118 E. Fourth Street, Tucson, Arizona 85721, USA \\ 2. College of Optical Sciences, University of Arizona, 1630 E. University Boulevard, Tucson, Arizona 85721, USA \\ 3. Opticslah, Albuquerque, New Mexico, USA \\ 4. Pacific Northwest National Laboratory, Richland, Washington 99352 USA \\ rjjones@optics.arizona.edu
}

\begin{abstract}
We characterize the dynamics of laser-induced plasmas using time-resolved dual-frequency-comb spectroscopy. The temporal evolution of plasma's temperature, population number density are estimated for multiple Fe transitions. (ㅇ) 2018 The Author(s)
\end{abstract}

OCIS codes: (140.0140) Lasers and laser optics, (280.5395) Plasma diagnostics, (350.5400) Plasmas.

Dual-frequency-comb spectroscopy offers time-resolved, broad bandwidth and high resolution measurements and has become a powerful tool in spectroscopic characterization. Laser-induced plasmas provides a useful means to standoff solid materials analysis. It has a wide spectrum of applications, ranging from isotope monitoring to planetary exploration. Emission spectroscopy and absorption spectroscopy are two major ways to characterize laser-induced plasmas, but each has its own limitations. Emission spectroscopy, such as laser-induced breakdown spectroscopy (LIBS) has an intrinsic trade-off between bandwidth and spectral resolution. Furthermore, emission spectroscopy is greatly affected by self-absorption, related to medium size and shape. As a result, complex numerical models [1] have to be used to get plasma information out from traditional LIBS measurement. On the other hand, absorption spectroscopy, such as laser absorption spectroscopy (LAS) [2] or laser-induced fluorescence (LIF), often cooperates with a tunable CW laser with high resolution. Limited by its scan speed and scan range, it is not ideal for broadband time-resolved measurements. Our previous work [3] has shown a proof of concept experiment for the first time that uses dual-comb spectroscopy to measure laser-induced plasmas, which combines the strengths of both broadband capability of LIBS and high spectral resolution of LAS. Here we fully utilize the high time-resolution of dual-comb spectroscopy to extract the time-resolved quantities (temperature, population density) of laser-induced plasmas.

Fig. 1 shows time-resolved transmittance of $3 \mathrm{Fe}$ I transitions around $533 \mathrm{~nm}$ using a stainless steel sample. $20 \mu$ s time-resolution is achieved with Blackman apodization window applied onto interferogram. The delay between plasma ablation and the center burst of interferogram varies from $11 \mu \mathrm{s}$ to $200 \mu \mathrm{s}$, controlled by a field programmable gate array (FPGA). Two frequency combs are homemade Yb fiber lasers with $f_{\text {rep }} \sim 78 \mathrm{MHz}$. Repetition rate difference is $\sim 500 \mathrm{~Hz}$. The repetition rates are measured individually, mixed together, low pass filtered, then seeded into a FPGA to trigger a Nd:YAG laser $\sim 10 \mathrm{~Hz}$ to ablate the stainless steel inside a vacuum chamber back filled with 100 Torr argon. A $12.5 \mathrm{~cm}$-focus-length lens is used to focus Nd:YAG laser to the sample. The focus spot size is $\sim 1 \mathrm{~mm}$. The dual-comb probe beam locates $0.83 \mathrm{~mm}$ above the sample. For each delay in Fig. 1, interferograms from 800 ablation shots are recorded using a 14-bit data acquisition card with $125 \mathrm{MS} / \mathrm{sec}$ sample rate, averaged in frequency domain after Fourier transformation. The background signals are recorded before ablation for each shot. The strength of each transition evolves over time. For instance, the strongest transition in the middle in Fig. 1 increases then decreases because as the plasma cools down, the majority of population shifts towards lower energy states. The relative strength of those 3 transitions is related to the population of lower levels of each transition. Together with Boltzmann distribution and partition function, we estimate the temperature and population number density.

Fig. 2 demonstrates absorption excitation temperature, total number density times interaction length, and lower energy level population times interaction length. For each transition, the integral of absorbance over frequency is proportional to corresponding lower level population when upper level population is negligible compared with that of lower level. Assuming thermal equilibrium, Boltzmann distribution links population with temperature. All 


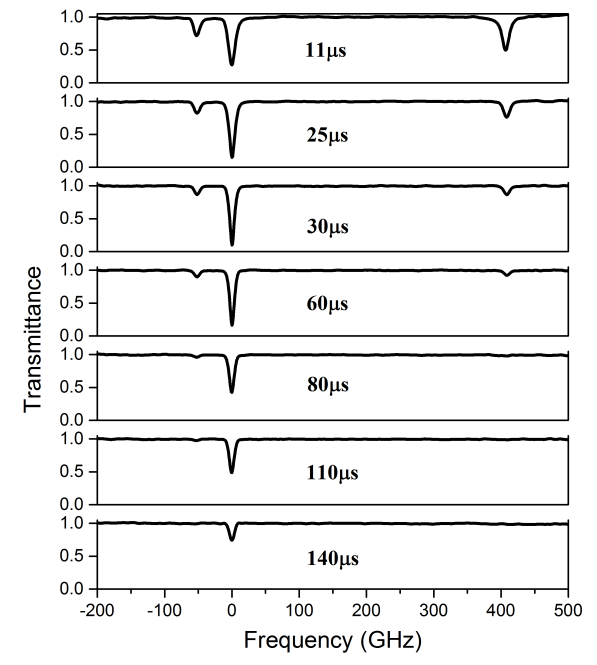

Fig. 1: Transmittance spectra of Fe I around $533 \mathrm{~nm}$ at different delays at which plasma is probed. It is plotted with optical frequency relative to the strongest center transition. The relative strength of these transitions indicates the population of each state, such that temperature can be found based on Boltzmann distribution.

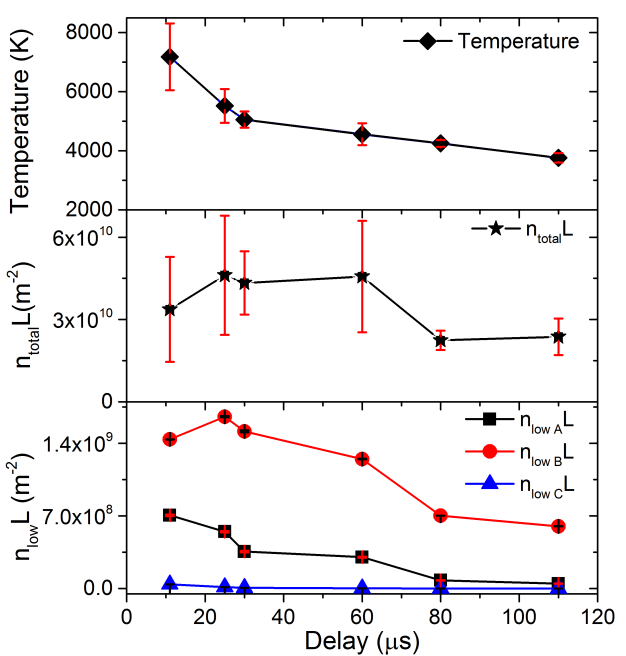

Fig. 2: From Fig. 1, absorption excitation temperature, total number density within interaction volume times interaction length and population of lower level of each transitions times interaction length are estimated. In Fig. 1, transitions are labeled as "A", "B", "C" from left to right.

together,

$$
\ln \left(\frac{\mathbf{A}_{\mathbf{i}}}{\sigma_{\mathbf{0}} \mathbf{g}_{\mathbf{i}} \mathbf{f}_{\mathbf{i j}}}\right)=\left(-\frac{1}{k_{B} T}\right) \cdot \mathbf{E}_{\mathbf{i}}+\ln \left(\frac{L \cdot n_{t o t}}{\mathbb{Z}(T)}\right)
$$

where $A_{i}$ is the area of absorbance of one transition, which provides $n_{l o w} L ; \sigma_{0}$ is the integrated absorption cross section $2.654 \times 10^{-6} \mathrm{~m}^{2} / \mathrm{s} ; f_{i j}$ is the oscillator strength; $g_{i}$ is degeneracy of level $i ; k_{B}$ is the Boltzmann constant; $Z(T)$ is the partition function; $E_{i}$ is the energy of lower state of a transition. Each of those three transitions contributes to one point in the plot of $\ln \left(\frac{A_{i}}{\sigma_{0} g_{i} f_{i j}}\right)$ versus $E_{i}$ for each delay. With linear fitting, the slope and intercept yield to temperature T and number density $n_{t o t} L$ at each delay. In the future, with a CCD camera to identify interaction length $L$, the absolute number density could be obtained, which is hard to access with traditional emission and absorption spectroscopy techniques with limited bandwidth.

Acknowledgements This material is based upon work supported by the Air Force Office of Scientific Research under award number FA9550-15-1-0091 and the Defense Threat Reduction Agency under Grant No. HDTRA 11710030. The Pacific Northwest National Laboratory is operated for the U.S. Department of Energy (DOE) by the Battelle Memorial Institute under Contract No. DE-AC05-76RL01830.

\section{References}

1. T. Sakka, et al., "Spatial population distribution of laser ablation species determined by self-reversed emission line profile" J. Appl. Phys. 92, 2296 (2002).

2. N. R. Taylor and M. C. Phillips, "Differential laser absorption spectroscopy of uranium in an atmospheric pressure laser-induced plasma" Opt. Lett. 39, 594 (2014)

3. J. Bergevin, T.-H. Wu, et al., "Dual comb spectroscopy of laesr-induced plasmas" Nature Comm. 9, 1273 (2018) 